\title{
Association of Tongue Bacterial Flora and Subtypes of Liver-Fire Hyperactivity Syndrome in Hypertensive Patients
}

\author{
Jie-wei Luo ${ }^{1},{ }^{1,2}$ Cong-huai Lin, ${ }^{2}$ Yao-bin Zhu, ${ }^{1,3}$ Xing-yu Zheng, ${ }^{1,2}$ \\ Yong-xi Wu, ${ }^{1,2}$ Wei-wei Chen, ${ }^{2}$ and Xiao Yang ${ }^{4}$ \\ ${ }^{1}$ Provincial Clinical Medical College, Fujian Medical University, Fuzhou 350001, China \\ ${ }^{2}$ Department of Traditional Chinese Medicine, Fujian Provincial Hospital, Fuzhou 350001, China \\ ${ }^{3}$ Department of Traditional Chinese Medicine, The First Affiliated Hospital, Fujian Medical University, Fuzhou 350005, China \\ ${ }^{4}$ Teaching and Research Office of Medical Cosmetology, Department of Management, Fujian Health College, Fuzhou 350101, China
}

Correspondence should be addressed to Jie-wei Luo; docluo0421@aliyun.com

Received 10 July 2017; Accepted 4 December 2017; Published 10 January 2018

Academic Editor: Salvatore Chirumbolo

Copyright (C) 2018 Jie-wei Luo et al. This is an open access article distributed under the Creative Commons Attribution License, which permits unrestricted use, distribution, and reproduction in any medium, provided the original work is properly cited.

\begin{abstract}
Structural changes in symbiotic human microorganisms can affect host phenotype. Liver-fire hyperactivity syndrome (LFHS) presents as bitter taste, halitosis, xerostomia, odontalgia, and other oral symptoms. LFHS is associated with hypertension (EH). In this study, tongue flora was analyzed to further understand the intrinsic relationship between tongue flora and LFHS. Samples of tongue coating, from 16 patients with EH-LFHS, 16 with EH-non-LFHS, and 16 controls, were obtained; then, 16S rRNA variable (V3-V4) regions were amplified and sequenced by MiSeq PE300 Sequencing. Tag clustering and Operational Taxonomic Units (OTUs) abundance analysis were used to compare the OTU sequence with the $16 \mathrm{~S}$ database. The species were classified, and diversity and structure of the bacterial flora were compared between the three groups. Alpha diversity analysis, including Observed Species index and Chao index, indicated significantly higher richness of species in patients with EH-LFHS $(p<0.05)$. Higher phylogenetic diversity, in patients with EH-non-LFHS, indicates greater differences in evolutionary history than in patients with EH-LFHS. Streptococcus, Rothia, Neisseria, and Sphingomonas were the most prevalent in patients with EH-LFHS, differed from the other two groups. This indicates that richer bacterial diversity, and structure associated with EH-LFHS, may affect the occurrence, development, and outcome of hypertension and syndrome subtypes recognized by TCM.
\end{abstract}

\section{Introduction}

The human microbiome refers to the collective genomes of microorganisms (symbiotic and pathogenic) that reside in and on the human body [1]. The microbiome is fundamentally important to the metabolism of the body. Within the past decade, programs to better understand the human microbiome in general, and more specifically the oral microbiome, have included the Human Microbiome Project (HMP) of the United States National Institutes of Health (NIH) [2] and the Human Oral Microbiome Database (HOMD) of the U.S. National Institute of Dental and Craniofacial Research (NIDCR) [3]. HOMD provides a detailed record of the type, metabolism, and pathogenicity of oral bacteria.

Traditional Chinese Medicine (TCM) has been practiced for over 5000 years. TCM relies on syndrome differentiation and treatment. Examination of the tongue is fundamentally important, because the tongue is considered a mirror of visceral changes that can predict an alteration in internal "Yin" and "Yang" of the body. In TCM, examination of the tongue provides an objective assessment of the state of habitus (strong or weak) and the rise and fall of vital Qi, which can be prognostic. The microbial flora, which coats the tongue, is formed by the Qi of the spleen and stomach. The coating on the tongue is normally thin and white, moderately wet, and the center and root of the tongue are a bit thick; this reflects the Qi of the stomach. Pathologies of the stomach can be reflected in the composition and appearance of the tongue flora [4].

The symbiotic microbiota of the human body has been termed "the second genome of the human body." 
Microorganisms are presumed to be the cause of variation that can affect the host phenotype [5]. According to TCM, the human and microbial genomes must be in harmonious coexistence to achieve the balance between Yin and Yang. The bacterial flora, of the coating of the tongue, is an important component of the oral flora, which significantly affects human health and is closely related to multisystem diseases.

Liver-fire hyperactivity syndrome (LFHS) presents as bitter taste, halitosis, xerostomia, odontalgia, and other oral symptoms. LFHS has been associated with hypertension [6, 7]. To better understand the intrinsic relationship between the flora of the tongue and liver-fire hyperactivity, we sequenced the oral flora of patients with LFHS, with and without essential hypertension (EH), as well as the flora of healthy individuals. For this, we used the U341F/U806R primer in the V3-V4 region from the variable region of 16sRNA. This approach broadly examines oral bacteria and archaea $[8,9]$.

\section{Materials and Methods}

2.1. Subjects. We recruited 32 patients, who had visited the Fujian Provincial Hospital from December 2014 to December 2015 and met the EH inclusion/exclusion criteria detailed below. These included 16 patients with $\mathrm{EH}$-liver-fire hyperactivity syndrome (EH-LFHS; 8 men and 8 women; average age $69.94 \pm 8.84$ years) and 16 patients with $\mathrm{EH}$-non-liverfire hyperactivity syndrome (EH-non-LFHS; 9 men and 7 women; average age $66.63 \pm 11.98$ years). Sixteen healthy control subjects, from the same hospital medical center, during the same period, were also enrolled $(7$ men and 9 women; average age $63.38 \pm 8.40$ years). The age and gender ratio of the three groups were comparable (both $p>0.1$ ).

2.2. Inclusion and Exclusion Criteria. All subjects were patients with hypertension without other significant diseases. The diagnostic criteria for hypertension were from the 2010 Chinese Guidelines for the Management of Hypertension [10]. Diagnostic criteria for liver-fire hyperactivity and EH-LFHS diagnostic criteria have been previously detailed [7]. The EH-LFHS diagnostic criteria included the main symptoms of dizziness, headache, and irritability. Secondary symptoms included flushing; ocular redness; dry mouth; bitter taste; constipation; dark urine with burning sensation; reddened tongue with thin and yellow fur; and wiry, rapid pulse. The exclusion criteria comprised presence of major diseases such as diabetes, coronary heart disease, heart failure, liver and kidney dysfunction, lung disease, tumor, stroke, or infection; pregnancy or lactation in women; smoking; and drinking.

2.3. Sample Acquisition and DNA Extraction. Samples of tongue coating were acquired as described in Version 12.0 of the procedure manual for the Human Microbiome Project (Accession: phd003190.2). Alcohol consumption and gargling were not allowed for the day preceding sampling. Patients fasted for 8 hours prior to sampling and did not brush their teeth on the day of sampling. For sampling, a $1 \mathrm{~cm}^{2}$ area at the center of the tongue was swabbed for 5 seconds using a Catch-All ${ }^{\mathrm{TM}}$ sample collection swab (Lucigen Corporation,
Middleton, WI, USA). Immediately after swabbing, the swab was swirled in $750 \mu \mathrm{L}$ of MoBio buffer in a MoBio tube. The swab sponge was pressed against the tube wall, multiple times for 20 seconds, to ensure the transfer of bacteria from the swab to the solution. After 30 minutes, the tube was transferred to an -80 centigrade freezer and stored until analysis.

For analysis, DNA was extracted, using a PowerSoil DNA isolation kit (Qiagen, Valencia, CA, USA), according to the manufacturer's instructions. PCR was conducted using a 16S rRNA universal primer 27F/1492R (27F: $5^{\prime}$-AGAGTTTGATCMTGGCTCAG-3'; 1492R: 5' -TACGGYTACCTTGTTACGACTT-3'; Thermo Fisher Scientific, Waltham, MA, USA).

2.4. Sequencing of the V3-V4 Region and $16 S$ rRNA. 16S specific primers were used to amplify the V3-V4 variable region. Amplification fragments of $425 \mathrm{bp}$ were obtained. Adaptor linkage was applied, and sequencing was conducted using a MiSeq PE300 apparatus (Illumina, San Diego, CA, USA). Paired-end reads, of $300 \mathrm{bp}$, were concatenated into longer tags using PANDAseq software as described previously [11]. Tag quality control included the following criteria: (1) no more than three ambiguous Ns on a tag; (2) restricted length between 300 and $500 \mathrm{bp}$; and (3) average base quality higher than Q20 (Kemp and Aller, 2004). Tags failing to satisfy these three requirements were discarded. Primers for sequencing bacterial 16S rDNA were as follows: U341: CCTACGGGRSGCAGCAG; U806: GGACTACVVGGGTATCTAATC $[8,9]$. Sequencing was conducted by Realbio Genomics Institute (Shanghai, China).

\subsection{Tag Clustering and Operational Taxonomic Unit (OTU)} Abundance. Tag sequences were checked for copy number. Tags, in which the sequences appeared at least twice, were considered reliable and were replicated. The replicated sequences were gathered into OTUs based on a softwareassessed similarity of 0.97 , and OTU-representing tags were chosen. All the tags of high quality were mapped on OTUrepresenting tags, with same similarity, to get the real abundance for each OTU. An OTU-to-sample abundance table was generated [12]. The database of the Ribosomal Database Project (RDP) was used to compare representative sequences with the 16S DNA of known species, and species classification was conducted for each OTU [13].

2.6. Diversity Analysis. Alpha diversity was conducted using Qiime python scripts. The purpose of alpha diversity is to determine the lowest number of tags necessary to achieve a plateau of rarefaction curves; therefore, all the tags were used. In the case of beta diversity, in which comparisons were made between samples, the tags of each sample were randomly downsized to the same number, even though we used relative abundances. Analyses were conducted using R statistical software (R Version 3.1.3).

2.7. Ethics Statement. This study was performed at the Fujian Provincial Hospital with the approval of the Medical Ethics Committee (K2014-005-01) of this hospital. Written informed consent was obtained from each patient. 


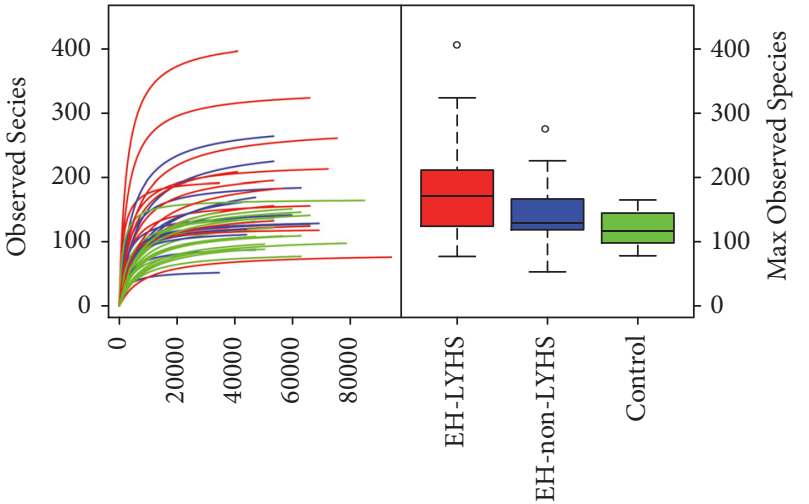

(a)

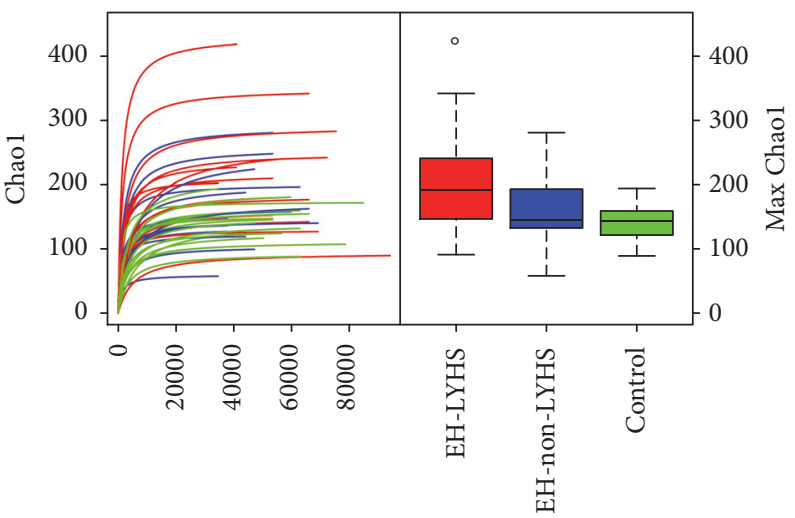

(b)

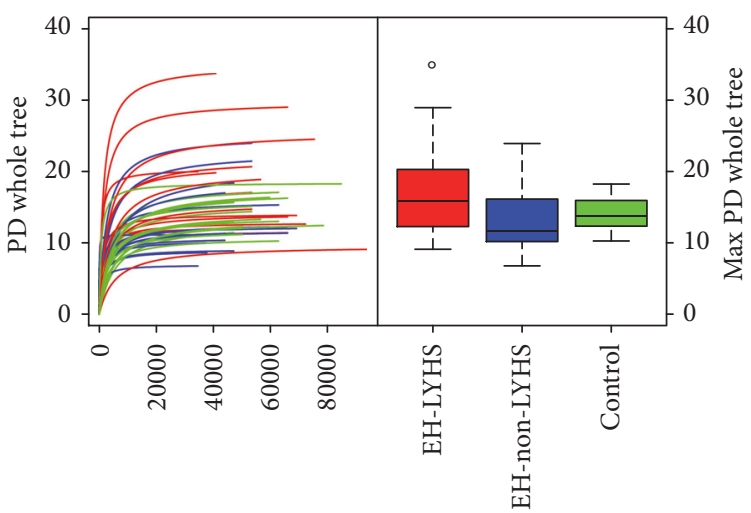

(c)

FIgure 1: Curves of the Observed Species index (a), Chao index (b), and PD whole tree index (c). In (a) and (b), the horizontal axis represents the number of clean reads randomly extracted from a sample, and the vertical axis indicates the alpha diversity index corresponding to the number of reads. Alpha diversity reflects the diversity of species in single samples including the Observed Species index, Chao index, and phylogenetic diversity (PD) whole tree index. Observed Species and Chao index reveal the richness of a community of species in the samples, regardless of the abundance of each species in the community. (c) The difference in the evolutionary history of species in a sample. The larger the PD whole tree index, the greater the difference of the species preserved in evolutionary history. A curve in the graph represents a sample. Outliers are marked with “o."

\section{Results}

3.1. Alpha Diversity and Drainage Analyses. Alpha diversity reflects the diversity of the species in individual samples including Observed Species index, Chao index, and phylogenetic diversity (PD) whole tree index [14]. Species richness data, of the Observed Species and Chao indices, revealed significantly greater richness in the EH-LFHS group than in the other two groups $(p<0.05)$. The PD whole tree index reflects the evolutionary history of the sample species. Compared with the EH-non-LFHS samples, the larger PD whole tree of the EH-LFHS samples (Figure 1 and Table 1) indicated a greater remaining difference of evolutionary history.

3.2. OTU. OTU abundance initially explained the abundance of species in the samples. The Kruskal test indicated that the clean reads, mapped reads, and final OTU of the EHLFHS group were significantly higher than those in the other two groups (all $p<0.05$; Table 2). Most of the samples appeared to be well sequenced, with 30000 or 34800 tags randomly picked for each sample. By contrast, the EH-LFHS group was most diverse and had more OTUs. As compared with the EH-non-LFHS group, the EH-LFHS group had more OTUs in common with the healthy controls. In this experiment, 992 different OTUs were obtained, and there were 231 significant OTU differences among the three groups $(p<0.05)$. The Principal Coordinates Analysis (PCoA), of the OTU abundance in each sample, indicated that there were significant differences in OTU abundance among the groups $(p<0.05)$ and that the three groups were well separated, according to OTU type, upon mapping (Figure 2(a)).

\subsection{Analysis of Sample Complexity and Significant Differences.} The species-profiling histogram can directly determine the specific species and their abundance, in a sample. The profiling histograms of the former top 10 species in each sample, at the genus level, are shown in Figure 3. The relationships among species are mainly symbiotic and antagonistic, and interspecific relationships can affect the abundance of the species. Figure 4 shows the strength of symbiotic and antagonistic relationships among the various genera. PCoA analysis indicated that samples were separated by groups at 
TABLE 1: Statistical results of alpha diversity of the groups.

\begin{tabular}{lccc}
\hline Group & Chao index & Observed Species index & PD whole tree index \\
\hline Liver-fire hyperactivity group & $191.5(144.75-241.5)$ & $170.5(123.5-212.75)$ & $16.5(12.25-20.75)$ \\
Non-liver-fire hyperactivity group & $145(130.25-195)$ & $129.5(117.75-168.25)$ & $12.5(10.25-16.75)$ \\
Healthy control group & $143.5(119.75-159.5)$ & $116.5(97.5-145.75)$ & $14.5(13-16.75)$ \\
Chi square value & 7.561 & 7.839 & 5.003 \\
$p$ value & 0.023 & 0.02 & $0.082^{\mathrm{a}}$ \\
\hline
\end{tabular}

${ }^{a}$ Liver-fire hyperactivity group versus non-liver-fire hyperactivity group; $p<0.05$.

TABLE 2: Statistical results of clean reads, mapped reads, and final OTU of each group.

\begin{tabular}{lccc}
\hline Group & Clean reads & Mapped reads & Final OTU \\
\hline Liver-fire hyperactivity group & $83956(69233-105696)$ & $57093(44645-69015)$ & $163(121-204)$ \\
Non-liver-fire hyperactivity group & $70694(60496-89226)$ & $48645(41072-63836)$ & $123(114-159)$ \\
Healthy control group & $78274(72718-90787)$ & $57515(49859-65527)$ & $114(87-136)$ \\
Chi square value & 2.576 & 3.113 & 8.824 \\
$p$ value & 0.276 & 0.211 & 0.012 \\
\hline
\end{tabular}

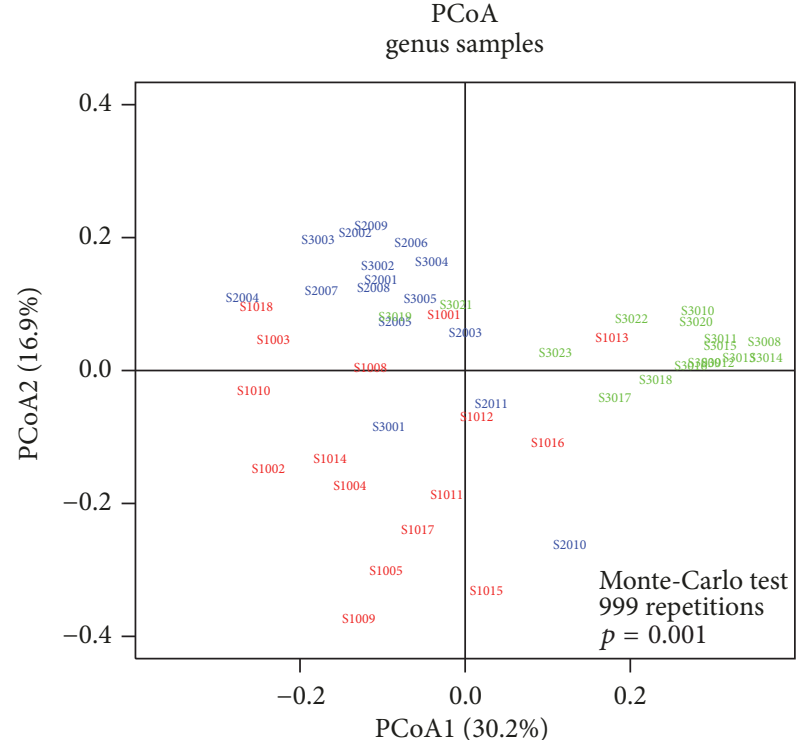

- EH-LYHS

- EH-non-LYHS

- Control

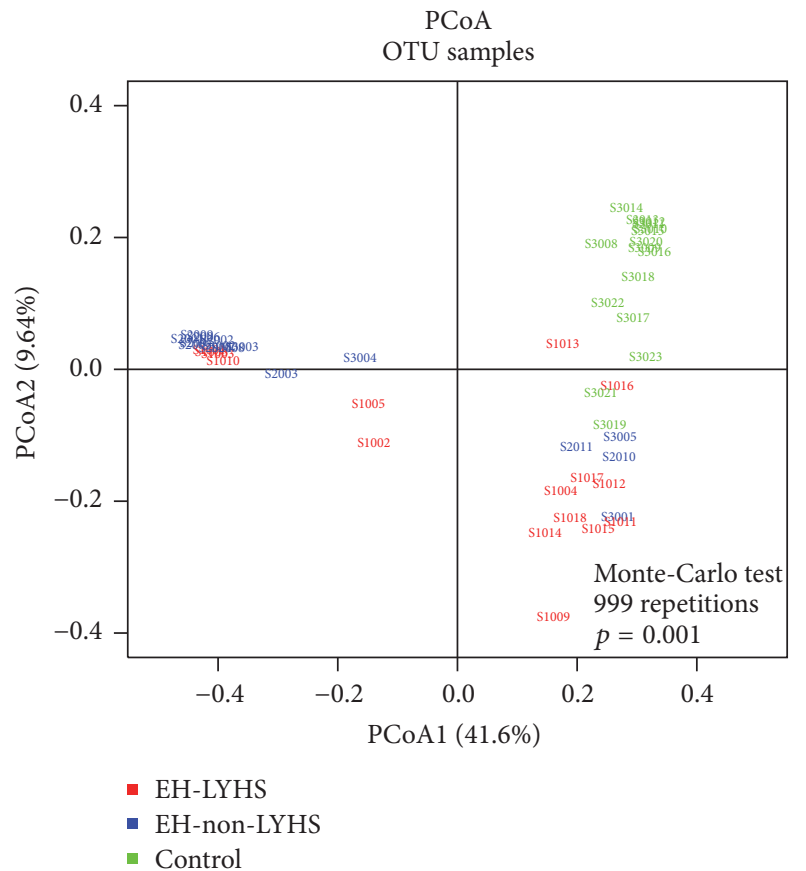

(b)

FIgURE 2: Principal Coordinates Analysis (PCoA) charts of OTU and genus samples. PCoA analysis reflects a dimensionality reduction and is a technique for analyzing and simplifying data sets. If the two samples are closer in distance, the compositions of the two samples will be more similar.

the genus level (Figure 2(b)). This suggests that disease status was the main factor in the significantly different bacterial composition $(p<0.05)$. Hence, it is likely that the diversity of microorganisms coating the tongue was strongly related to EH-LFHS and EH-non-LFHS.

3.4. Linear Discriminant Analysis (LDA). The LDA effect size (LEfSe) tools are used to estimate how the abundance of each component (species) affects the differences between classes or groups; these tools are also used to identify communities or species having significant differences in sample classification [15]. The LDA distribution histograms of each group are presented in Table 3. The length of the histogram represents the extent of the influence of significant difference species. The evolutionary cladogram, shown in Figure 5, visually represents the major species, which result in differences among each group. The results indicate the different bacterial composition between the groups at the genus level. Samples, acquired from healthy controls, were abundant in Veillonella, while Prevotella was prevalent in the EH-non-LFHS group. 


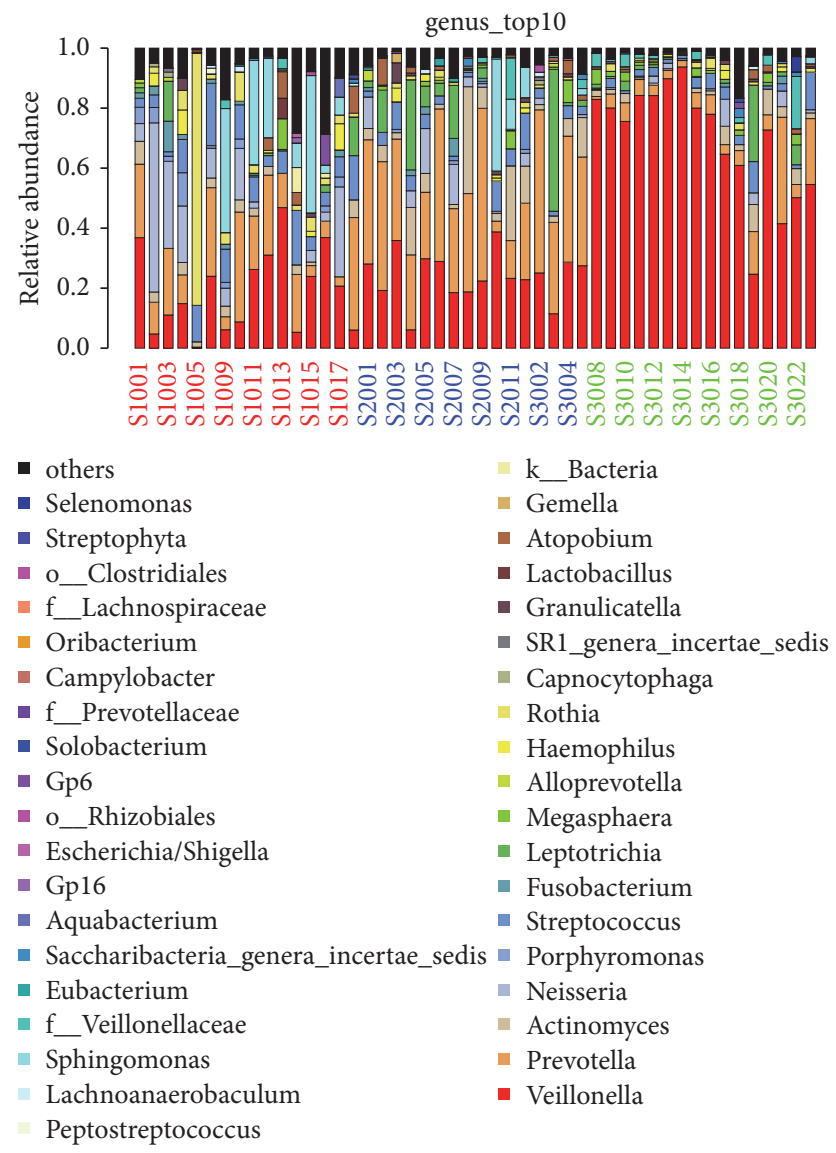

FIGURE 3: The profiling histogram of the former top 10 species in each sample at the genus level.

The compositions of the samples, acquired from the EH-nonLFHS and control groups, were similar, while that of the EHLFHS group was relatively more varied. At the phylum level, the difference between the EH-LFHS and EH-non-LFHS groups mainly involved Proteobacteria and Fusobacteria; EHLFHS samples contained more Proteobacteria. The prevalence of Firmicutes, Bacteroidetes, and Actinobacteria was similar. In the healthy controls, Firmicutes predominated. The three groups differed significantly in 54 genera. In addition to Veillonella and Prevotella (Table 3), Actinomyces was most abundant in the EH-non-LFHS group. The prevalence of Porphyromonas, Rothia, Parvimonas, and Streptococcus was greater in the EH-LYHS group. Megasphaera was somewhat more prevalent in healthy controls.

\section{Discussion}

Disruption of microbial homeostasis can produce clinical symptoms. Microbial stability is correlated with the abundance of relevant tissues. For example, irritable bowel syndrome and inflammatory bowel disease are closely related to the amount of intestinal Bifidobacterium [16, 17]. Host genetics are also important; for example, African American women are more susceptible to bacterial vaginosis than nonHispanic white women [18]. Overuse of antibiotics may

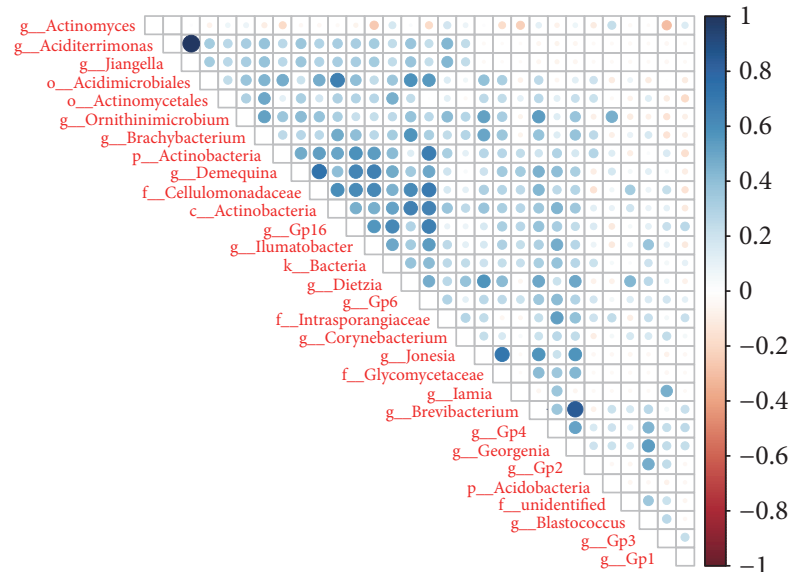

FIGURE 4: Symbiosis and antagonism of various fungi at the genus level. At the genus level, the strength of symbiotic and antagonistic relationships was obtained by rank sum test, and there was a correlation among the top 30 differentiated species. The blue on the right shows a positive correlation, and the red shows a negative correlation. The darker the color, the stronger the correlation between the species.

cause the translocation of intestinal bacteria and disrupt the stability of microbial communities [19]. Diet is another factor that affects the stability of human microbes. For example, the abundance of gingival anaerobic bacteria decreases more in people who consume coffee and wine than in those who do not; these differences in the microbial population can affect oral health [20].

How human symbiotic bacteria cause disease remains unclear. An imbalance in the intestinal flora may result in metabolic syndrome, peptic ulcer, heart disease, and obesity. Dysregulation of microbial molecules may directly affect the permeability of the intestinal epithelium; via the pituitary adrenal axis (HPA axis), it may also induce inflammatory responses and insulin resistance [21, 22]. In addition, intestinal microflora, such as segmental filamentous bacteria, Bacteroides, and spindle cells, can promote the differentiation of $\mathrm{T}$ cells, resulting in strong innate and adaptive immune responses in the intestine [23]. Intestinal microflora may affect mood and behavior through a complex brain-gut axis; for example, decreased numbers of intestinal Clostridium species have been linked to impaired stress response in mice [24]. Recent research on plasma oxidation of trimethylamine $\mathrm{N}$-oxide (TMAO) has shown that degradation of TMAO can decrease the level of cholesterol in the blood, increasing the risk of atherosclerosis, coronary heart disease, heart failure, and other cardiovascular disorders $[25,26]$. Foods, rich in choline or trimethylamine, are the basis of TMAO, which is generated by the action of intestinal microbial degradative enzymes that can rapidly enter the liver through blood. The reaction also involves flavin monooxygenases. TMAO is eventually removed via the kidneys [27, 28]. Although these studies examined the pathogenicity of intestinal microbial disorders, it is possible that metabolites from oral flora can exert similar effects. 

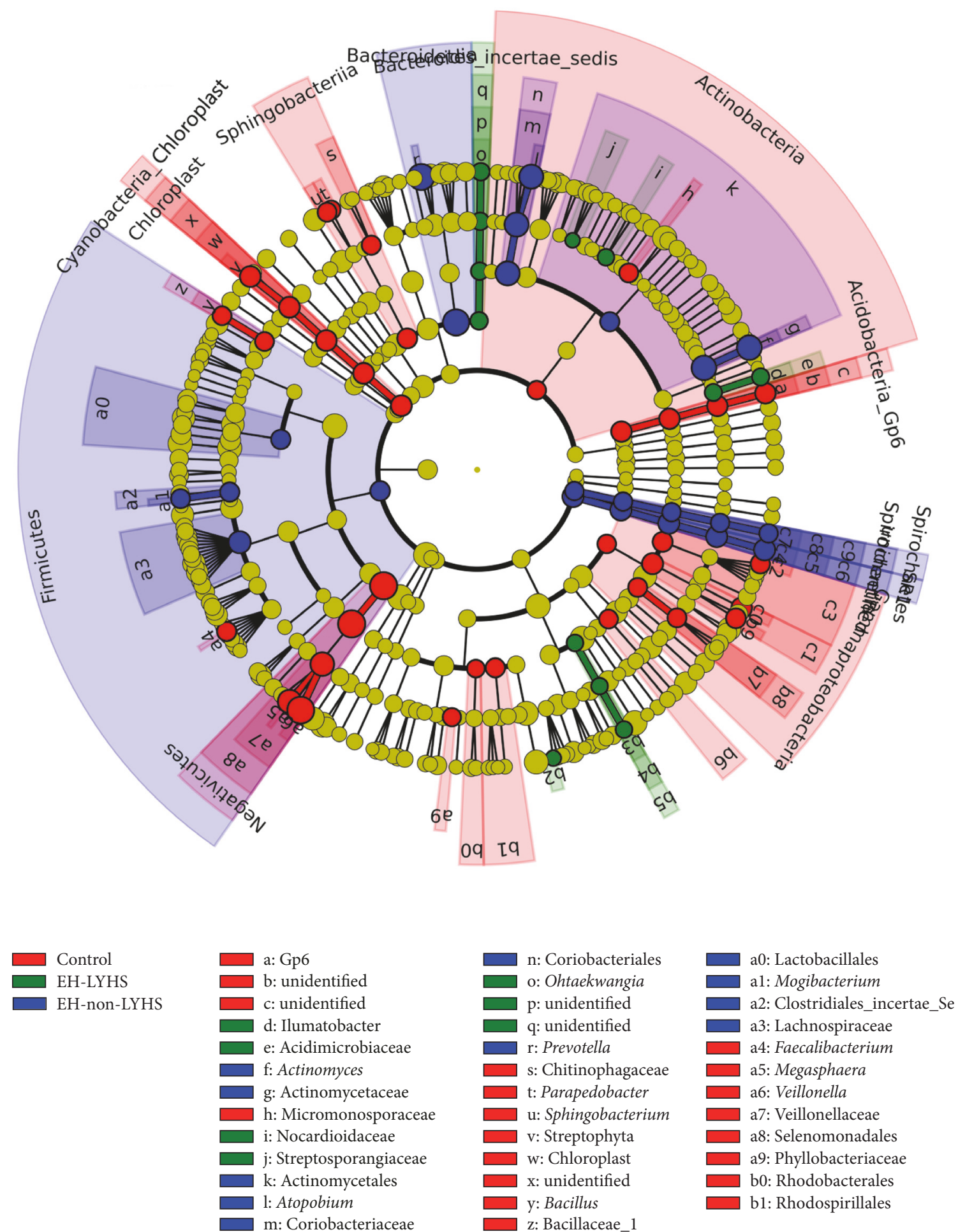

FIGURE 5: Evolutionary bifurcation diagram of LDA effect size. The evolutionary bifurcation diagram can visually represent the major species, indicating the differences in each group; inner to outer circles represent the taxonomic level from the phylum to the genus. Each small circle, at different classification levels, represents a classification under this level; the diameter of the small circle is proportional to relative abundance. 
TABLE 3: Species with significant differences in abundance in EH-non-LYHS, EH-non-LYHS, and control group, as assessed by Linear Discriminant Analysis (LDA).

\begin{tabular}{|c|c|c|c|}
\hline $\begin{array}{l}\text { LDA score }(\log \\
10)\end{array}$ & $\begin{array}{l}\text { Liver-fire hyperactivity } \\
\text { group }\end{array}$ & $\begin{array}{l}\text { Non-liver-fire hyperactivity } \\
\text { group }\end{array}$ & Healthy control group \\
\hline $8<$ score $\leqslant 10$ & & $\begin{array}{l}\text { Bacteroidia }>\text { Prevotella }> \\
\text { Actinomycetaceae }> \\
\text { Actinomyces }\end{array}$ & Selenomonadales $>$ Negativicutes $>$ Veillonella \\
\hline $6<$ score $\leqslant 8$ & $\begin{array}{l}\text { Streptosporangiaceae }> \\
\text { Achromobacter }> \\
\text { Nocardioidaceae> } \\
\text { unidentified }> \\
\text { Methylophilales> } \\
\text { Methylophilaceae> } \\
\text { Methylophilus }> \\
\text { unidentified }> \\
\text { Ilumatobacter> } \\
\text { Acidimicrobiaceae> } \\
\text { cteroidetes_incertae_sedis }> \\
\text { Ohtaekwangia }\end{array}$ & $\begin{array}{l}\text { Coriobacteriales }> \\
\text { Coriobacteriaceae> } \\
\text { Atopobium }> \\
\text { Lachnospiraceae> } \\
\text { SRl_genera_incertae_sedis } \\
>\text { SRl>unidentified }> \\
\text { unidentified }>\text { unidentified }> \\
\text { Mogibacterium }> \\
\text { ridiales_lncertae_Sedis_XI 11> } \\
\text { Actinomycetales }\end{array}$ & $\begin{array}{l}\text { Veillonellaceae }>\text { Megasphaera }> \\
\text { Gammaproteobacteria }>\text { Phyllobacteriaceae }> \\
\text { Actinobacteria }>\text { Sphingobacterium }> \\
\text { Myxococcales }>\text { Rhodobacterales }> \\
\text { Parapedobacter }>\text { Micromonosporaceae }> \\
\text { Stenotrophomonas }>\text { Pseudomonadales }> \\
\text { Faecalibacterium }>\text { Rhodospirillales }> \\
\text { Acinetobacter }>\text { Bacillaceae_1 }>\text { Bacillus }> \\
\text { Pseudomonas }>\text { Cyanobacteria_Chloroplast }> \\
\text { unidentified }>\text { Acidobacteria_Gp6 }>\text { Gp6 }> \\
\text { unidentified }>\text { unidentified }>\text { Streptophyta }> \\
\text { Chloroplast }>\text { Chloroplast }>\text { Sphingobacteriia }> \\
\text { Xanthomonadales }>\text { Chitinophagaceae } \\
\end{array}$ \\
\hline $4<$ score $\leqslant 6$ & & $\begin{array}{l}\text { Firmicutes }>\text { Lactobacillales }> \\
\text { Treponema }>\text { Spirochaetia } \\
>\text { Spirochaetes }> \\
\text { Spirochaetaceae }> \\
\text { Spirochaetales }\end{array}$ & Enterobacteriales $>$ Enterobacteriaceae \\
\hline
\end{tabular}

Note. LDA score $(\log 10)$ represents the magnitude of the effect in species with significant differences in abundance.

Accumulating evidence indicates that oral microbiota is related to periodontal, cardiovascular, and other diseases. An imbalance in microecology may initiate or promote the process of atherosclerosis [29, 30]. Murine macrophages have been cocultured with $P$. gingivalis in the presence of lowdensity lipoproteins (LDL); this has been shown to promote LDL mobility and lead to dose-dependent formation of foam cells, caused in part by LDL aggregation via proteolysis of ApoB-100 [31]. The formation of foam cells is widely recognized as the initial critical step in atherosclerosis and atherosclerosis markers. Studies using heterozygous apolipoprotein E-deficient $[\operatorname{ApoE}(+/-)]$ mice, fed a highfat diet or regular chow and inoculated or not inoculated with $P$. gingivalis, have shown the following. Proximal aortic lesion size, at 24 weeks, was 9-fold greater in the chowfed mice inoculated with $P$. gingivalis than in noninoculated mice and was 2 -fold greater in $P$. gingivalis-inoculated versus noninoculated high-fat diet-fed mice. In another study, atherosclerotic plaques were rich in macrophages, and $P$. gingivalis ribosomal DNA was found in the aorta, heart, and liver [32]. Oxidation of LDL is mediated by external factors; the above studies suggest that $P$. gingivalis can induce LDL oxidation, enhance vascular oxidative stress, and promote the development of atherosclerosis. Do other oral bacteria have the same effect? Periodontal bacteria have been detected in infected heart valves and aortic aneurysms [33-35]. Chryseomonas, Wei Rong Staphylococcus, and Streptococcus were found more prevalent in the oral flora of 15 people with atherosclerosis than in that of healthy individuals; bacterial DNA was also found in the atherosclerotic plaques of the individuals with atherosclerosis [36]. The abundance of Wei Rong Staphylococcus and Streptococcus in plaque may be related to the abundance of oral white blood cells and the level of plasma cholesterol.

Intestinal flora may be an important environmental factor in hypertension [37]. An imbalance in oral flora may also affect the regulation of blood pressure. In one study, 15 hypertensive volunteers were randomly allocated to the experimental group and control group. The experimental group rinsed their mouths with an antiseptic mouthwash, while the control group used sterile water. After 3 consecutive days, the average systolic blood pressure of the experimental group increased by $2.3 \mathrm{mmHg}$, while the diastolic blood pressure did not change; oral nitrate reduction in the experimental group was decreased. The levels of nitrite in saliva decreased significantly, while those of nitrate increased; plasma nitrite, in the experimental group, had also decreased [38].

Nitric oxide (NO) is a lipophilic gas. It is a protective factor in the cardiovascular system and plays a key role in maintaining vascular tone and blood pressure. There are two main sources of intravascular NO. Using L-arginine as substrate, NADPH and L-arginine are oxidized to Lcitrulline and NO nicotinamide adenine dinucleotide phosphate (NADP) by the action of nitric oxide synthase (NOS). In addition, dietary nitrate is decomposed to nitrite and NO with the help of oral microorganisms, suggesting that oral microorganisms can affect cardiovascular disease in a variety of ways.

In our study, the abundance of Veillonella in the tongue coating of healthy controls was higher than that in patients 
with hypertension. In normal flora, Veillonella in the human natural cavity tract is involved with the formation of plaque biofilm and can reduce dental caries. Streptococcus, the predominant bacterium associated with EH-LFHS, is a grampositive bacterium, closely related to oral symptoms such as halitosis. Rothia are a gram-positive bacilli that can reduce nitrate. Neisseria are gram-negative cocci that are involved in the formation of plaque biofilms. Normal oral parasitic bacteria, such as Rothia and Neisseria, may be conditional pathogens in endocarditis $[39,40]$. Sphingomonas is gramnegative bacterium, which can convert pentose, hexose, and disaccharide into acids. There are few studies on it thus far. Prevotella and Bacteroides, present in the normal flora of the oral, gut, and female genital tract, predominated in the EHnon-LFHS group. Bacteroides is not highly pathogenic, and its abundance may also be related to oral symptoms.

Fifty-four bacterial genera differed between the three experimental groups, illustrating that differences in the composition of oral flora may be related to the absence or presence of hypertension. This suggests an association between the incidence of hypertension disorders and imbalance in the oral microbiome. The abundance of Porphyromonas, Rothia, Parvimonas, Streptococcus, and other bacteria, in the tongue coating of patients with EH-LFHS exhibiting symptoms such as halitosis and bitter taste, was significantly higher than that of the EH-non-LFHS and control groups. This indicates the presence of different flora in TCM syndromes. Volatile sulfide metabolites are produced by the decomposition of sulfur-containing amino acids by oral microorganisms. These metabolites include sulfurated hydrogen, methyl mercaptan, dimethyl mercaptan, benzopyrrole, ammonia, butyric acid, isovaleric acid, and other odorous gases. The biological diversity of the dorsal tongue flora, in patients with halitosis, is higher than that in individuals without halitosis. Leaf Bacillus, Veillonella, Solobacterium, and Moorei may be related to halitosis [41]. Increased proportion of oral anaerobic bacteria leads to the release of sulfur protein metabolites, in turn leading to halitosis and other oral symptoms $[42,43]$. The change in oral microflora may also affect cholesterol and bile acid metabolism in patients with hypertension, promoting the production of inflammatory factors and endothelial function damage; this plays a role in the pathogenesis of hypertension. Autonomic nerve dysfunction and internal environment disorders, caused by the disease itself, may also cause imbalances in oral flora, worsening the condition.

Therefore, applying TCM to examine the effects of the oral environment can be instrumental in the prevention and treatment of hypertension and the balance of Yin and Yang.

\section{Abbreviations}

TCM: $\quad$ Traditional Chinese Medicine

EH: $\quad$ Essential hypertension

EH-LFHS: $\quad$ EH-liver-fire hyperactivity syndrome

EH-non-LFHS: EH-non-liver-fire hyperactivity syndrome

LFHS: Liver-fire hyperactivity syndrome

OTUs: Operational Taxonomic Units

TMAO: $\quad$ Trimethylamine N-oxide

TMA: Trimethylamine
FMOs: Flavin monooxygenase

LDL: Low-density lipoprotein

NO: Nitric oxide

NOS: Nitric oxide synthase

NADP: Nicotinamide adenine dinucleotide phosphate.

\section{Conflicts of Interest}

The authors declare that they have no conflicts of interest.

\section{Authors' Contributions}

Jie-wei Luo, Cong-huai Lin, and Yao-bin Zhu conceived and designed the experiments. Jie-wei Luo, Cong-huai Lin, Yaobin Zhu, Xing-yu Zheng, Yong-xi Wu, Wei-wei Chen, and Xiao Yang performed the experiments. Jie-wei Luo, Conghuai Lin, and Yao-bin Zhu analyzed the data and wrote the manuscript. All authors read and approved the final version of the manuscript. Jie-wei Luo, Cong-huai Lin, and Yao-bin Zhu contributed equally to this work.

\section{Acknowledgments}

This work was supported by the Financial Scheme for Young Talents Training Program of the Fujian Health Industry (Grant no. 2015-ZQN-ZD-7) and Fujian Provincial Health and Family Planning Youth Research Program (no. 2016-184), Fujian, China.

\section{References}

[1] J. Lederberg and A. T. McCray, “Ome sweet' omics - a genealogical treasury of words," The Scientist, vol. 15, no. 7, p. 8, 2001.

[2] NIH HMP Working Group, J. Peterson, S. Garges et al., "The NIH human microbiome project," Genome Research, vol. 19, no. 12, pp. 2317-2323, 2009.

[3] NIH/National Institute of Dental and Craniofacial Research, "First comprehensive database of human oral microbiome launched," ScienceDaily, 2008, https://www.sciencedaily.com/ releases/2008/03/080326121227.htm.

[4] T. F. Wang, Diagnostics of Traditional Chinese Medicine, pp. 25-33, People's Medical Publishing House, Beijing, China, 2nd edition, 2013.

[5] C. Moon, M. T. Baldridge, M. A. Wallace, C.-A. D. Burnham, H. W. Virgin, and T. S. Stappenbeck, "Vertically transmitted faecal IgA levels determine extra-chromosomal phenotypic variation," Nature, vol. 521, no. 7550, pp. 90-93, 2015.

[6] J. W. Luo, X. Chen, L. Ren, and X. M. Zhang, "A study on syndrome differentiation of hypertension complicated with oral symptoms," Fujian Journal of TCM, vol. 30, no. 9, pp. 12-13, 1999 (Chinese).

[7] X. Y. Zheng, The Principle of Clinical Research on New Drugs of Chinese Medicine (Trial), pp. 240-245, China Medical Science Press, Beijing, China, 2002.

[8] Y. Wang and P.-Y. Qian, "Conservative fragments in bacterial $16 S$ rRNA genes and primer design for $16 S$ ribosomal DNA amplicons in metagenomic studies," PLoS ONE, vol. 4, no. 10, Article ID e7401, 2009.

[9] M. Zakrzewski, A. Goesmann, S. Jaenicke et al., "Profiling of the metabolically active community from a production-scale 
biogas plant by means of high-throughput metatranscriptome sequencing," Journal of Biotechnology, vol. 158, no. 4, pp. 248258, 2012.

[10] The Revising Committee of Chinese Guidelines for Hypertension Prevention and Treatment, "2010 Chinese guidelines for the management of hypertension," Chinese Journal of Cardiology, vol. 39, no. 7, pp. 579-616, 2011 (Chinese).

[11] A. P. Masella, A. K. Bartram, J. M. Truszkowski, D. G. Brown, and J. D. Neufeld, "PANDAseq: paired-end assembler for illumina sequences," BMC Bioinformatics, vol. 13, p. 31, 2012.

[12] R. C. Edgar, "UPARSE: highly accurate OTU sequences from microbial amplicon reads," Nature Methods, vol. 10, no. 10, pp. 996-998, 2013.

[13] D. McDonald, M. N. Price, J. Goodrich et al., "An improved Greengenes taxonomy with explicit ranks for ecological and evolutionary analyses of bacteria and archaea," The ISME Journal, vol. 6, no. 3, pp. 610-618, 2012.

[14] P. F. Kemp and J. Y. Aller, "Bacterial diversity in aquatic and other environments: what $16 \mathrm{~S}$ rDNA libraries can tell us," FEMS Microbiology Ecology, vol. 47, no. 2, pp. 161-177, 2004.

[15] N. Segata, J. Izard, L. Waldron et al., "Metagenomic biomarker discovery and explanation," Genome Biology, vol. 12, no. 6, article R60, 2011.

[16] E. Malinen, T. Rinttilä, K. Kajander et al., "Analysis of the fecal microbiota of irritable bowel syndrome patients and healthy controls with real-time PCR," American Journal of Gastroenterology, vol. 100, no. 2, pp. 373-382, 2005.

[17] P. Seksik, L. Rigottier-Gois, G. Gramet et al., "Alterations of the dominant faecal bacterial groups in patients with Crohn's disease of the colon," Gut, vol. 52, no. 2, pp. 237-242, 2003.

[18] R. B. Ness, S. Hillier, H. E. Richter et al., "Can known risk factors explain racial differences in the occurrence of bacterial vaginosis?" Journal of the National Medical Association, vol. 95, no. 3, pp. 201-212, 2003.

[19] L. Dethlefsen and D. A. Relman, "Incomplete recovery and individualized responses of the human distal gut microbiota to repeated antibiotic perturbation," Proceedings of the National Acadamy of Sciences of the United States of America, vol. 108, supplement 1, pp. 4554-4561, 2011.

[20] C. Signoretto, F. Bianchi, G. Burlacchini, F. Sivieri, D. Spratt, and P. Canepari, "Drinking habits are associated with changes in the dental plaque microbial community," Journal of Clinical Microbiology, vol. 48, no. 2, pp. 347-356, 2010.

[21] V. Tremaroli and F. Bäckhed, "Functional interactions between the gut microbiota and host metabolism," Nature, vol. 489, no. 7415, pp. 242-249, 2012.

[22] E. A. Mayer, K. Tillisch, and A. Gupta, "Gut/brain axis and the microbiota," The Journal of Clinical Investigation, vol. 125, no. 3 , pp. 926-938, 2015.

[23] N. Cerf-Bensussan and V. Gaboriau-Routhiau, "The immune system and the gut microbiota: friends or foes?" Nature Reviews Immunology, vol. 10, no. 10, pp. 735-744, 2010.

[24] M. T. Bailey, S. E. Dowd, J. D. Galley, A. R. Hufnagle, R. G. Allen, and M. Lyte, "Exposure to a social stressor alters the structure of the intestinal microbiota: implications for stressor-induced immunomodulation," Brain, Behavior, and Immunity, vol. 25, no. 3, pp. 397-407, 2011

[25] Z. Wang, A. B. Roberts, J. A. Buffa et al., "Non-lethal inhibition of gut microbial trimethylamine production for the treatment of atherosclerosis," Cell, vol. 163, no. 7, pp. 1585-1595, 2015.
[26] J. L. Griffin, X. Wang, and E. Stanley, "Does our gut microbiome predict cardiovascular risk? A review of the evidence from metabolomics," Circulation: Cardiovascular Genetics, vol. 8, no. 1, pp. 187-191, 2015.

[27] Z. Wang, E. Klipfell, B. J. Bennett et al., "Gut flora metabolism of phosphatidylcholine promotes cardiovascular disease," Nature, vol. 472, no. 7341, pp. 57-63, 2011.

[28] J. Hartiala, B. J. Bennett, W. H. W. Tang et al., "Comparative genome-wide association studies in mice and humans for trimethylamine $\mathrm{N}$-Oxide, a proatherogenic metabolite of choline and L-carnitine," Arteriosclerosis, Thrombosis, and Vascular Biology, vol. 34, no. 6, pp. 1307-1313, 2014.

[29] J. T. Katz and R. P. Shannon, "Bacteria and coronary atheroma: More fingerprints but no smoking gun," Circulation, vol. 113, no. 7, pp. 920-922, 2006.

[30] J. H. Meurman, M. Sanz, and S.-J. Janket, "Oral health, atherosclerosis, and cardiovascular disease," Critical Reviews in Oral Biology and Medicine, vol. 15, no. 6, pp. 403-413, 2004.

[31] H. Miyakawa, K. Honma, M. Qi, and H. K. Kuramitsu, "Interaction of porphyromonas gingivalis with low-density lipoproteins: implications for a role for periodontitis in atherosclerosis," Journal of Periodontal Research, vol. 39, no. 1, pp. 1-9, 2004.

[32] L. Li, E. Messas, E. L. Batista Jr., R. A. Levine, and S. Amar, "Porphyromonas gingivalis infection accelerates the progression of atherosclerosis in a heterozygous apolipoprotein E-deficient murine model," Circulation, vol. 105, no. 7, pp. 861-867, 2002.

[33] K. Nakano, H. Inaba, R. Nomura et al., "Distribution of Porphyromonas gingivalis fimA genotypes in cardiovascular specimens from Japanese patients," Oral microbiology and immunology, vol. 23, no. 2, pp. 170-172, 2008.

[34] K. Nakano, H. Nemoto, R. Nomura et al., "Detection of oral bacteria in cardiovascular specimens," Oral microbiology and immunology, vol. 24, no. 1, pp. 64-68, 2009.

[35] N. Kurihara, Y. Inoue, T. Iwai, M. Umeda, Y. Huang, and I. Ishikawa, "Detection and localization of periodontopathic bacteria in abdominal aortic aneurysms," European Journal of Vascular and Endovascular Surgery, vol. 28, no. 5, pp. 553-558, 2004.

[36] O. Koren, A. Spor, J. Felin et al., "Human oral, gut, and plaque microbiota in patients with atherosclerosis," Proceedings of the National Acadamy of Sciences of the United States of America, vol. 108, Supplement 1, pp. 4592-4598, 2011.

[37] H. Jin, Z. Jin, and L. L. Zhang, "Gut microbiota might be an environmental factor in the development of essential hypertension," Chinese Journal of Microecology, vol. 27, no. 1, pp. 121-125, 2015.

[38] C. P. Bondonno, A. H. Liu, K. D. Croft et al., "Antibacterial mouthwash blunts oral nitrate reduction and increases blood pressure in treated hypertensive men and women," American Journal of Hypertension, vol. 28, no. 5, pp. 572-575, 2015.

[39] S. Shakoor, N. Fasih, K. Jabeen, and B. Jamil, "Rothia dentocariosa endocarditis with mitral valve prolapse: Case report and brief review," Infection, vol. 39, no. 2, pp. 177-179, 2011.

[40] J. E. Carter, K. N. Mizell, and T. N. Evans, "Neisseria sicca meningitis following intracranial hemorrhage and ventriculostomy tube placement," Clinical Neurology and Neurosurgery, vol. 109, no. 10, pp. 918-921, 2007.

[41] J. Wang, M. R. Wang, and Z. L. Wang, "Diversity of bacterial population on the tongue dorsa of halitosis patients," Beijing Journal of Stomatology, vol. 18, no. 1, pp. 25-41, 2010. 
[42] T. Takeshita, N. Suzuki, Y. Nakano et al., "Discrimination of the oral microbiota associated with high hydrogen sulfide and methyl mercaptan production," Scientific Reports, vol. 2, p. 215, 2012.

[43] T. Takeshita, N. Suzuki, Y. Nakano et al., "Relationship between oral malodor and the global composition of indigenous bacterial populations in saliva," Applied and Environmental Microbiology, vol. 76, no. 9, pp. 2806-2814, 2010. 


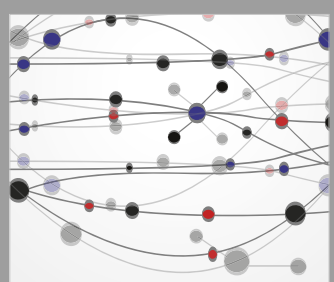

The Scientific World Journal
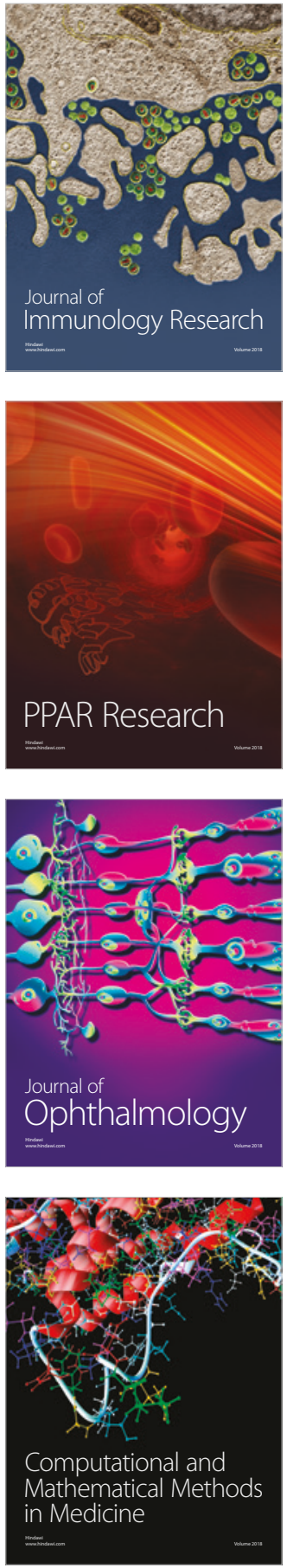

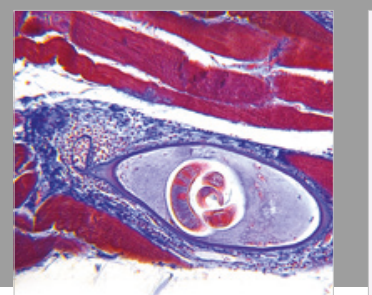

Gastroenterology Research and Practice

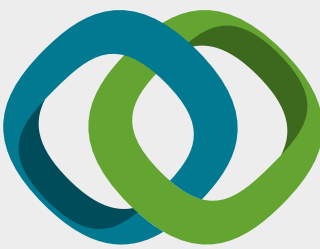

\section{Hindawi}

Submit your manuscripts at

www.hindawi.com
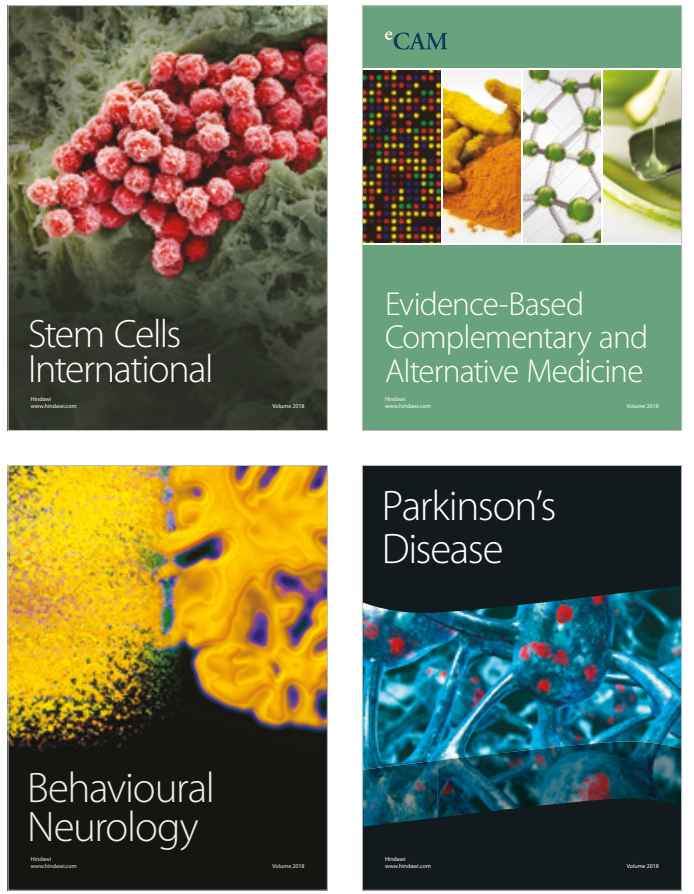

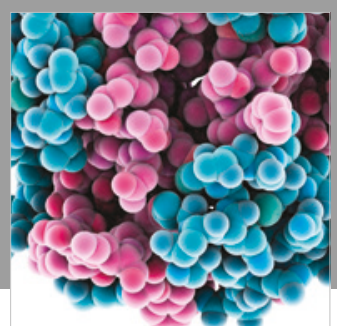

ournal of

Diabetes Research

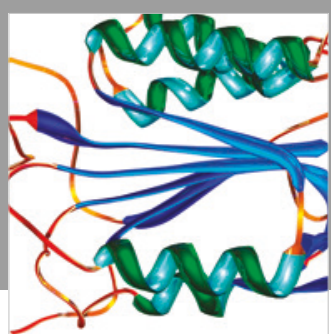

Disease Markers
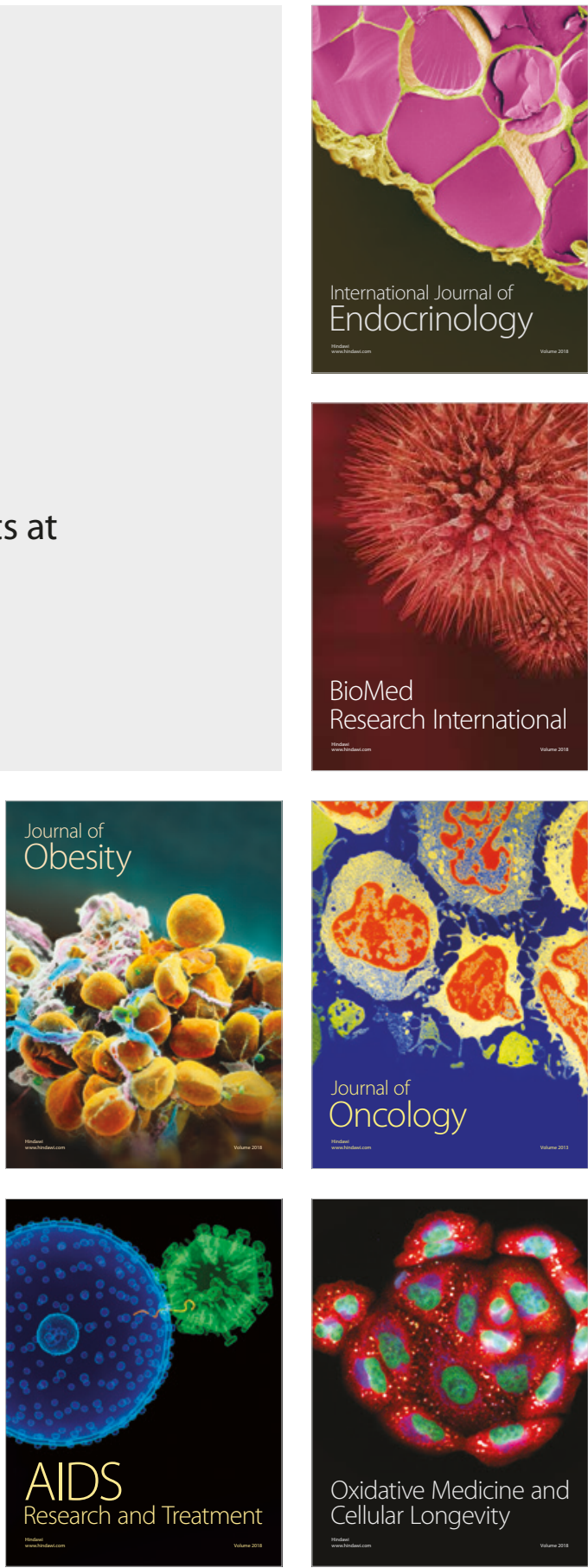